\title{
LES LYMPHOMES DE L'ANNEAU DE WALDEYER TRAITEMENT ET PRONOSTIC
}

\author{
M. BELCADHI, R. FDHILA, H. EL KHLIFI, H. CHAHED, W. KERMANI, I. ZEGLAOUI, R. MANI, \\ M. BEN ALI, M. ABDELKEFI, H. EL OMRI ${ }^{*}$ B. SRIHA** K. BOUZOUITA \\ SERVICE D'ORL ET DE CHIRURGIE CERVICO-FACIALE \\ *SERVICE D'HÉMATOLOGIE CLINIQUE \\ ${ }^{*}$ 'SERVICE D'ANATOMIE PATHOLOGIQUE \\ HÔPITAL FARHAT HACHED, SOUSSE
}

\begin{abstract}
Les lymphomes non Hodgkiniens (LNH) qui prennent naissance au niveau de l'anneau de Waldeyer constituent une entité bien particulière tant sur le plan clinique, thérapeutique que pronostique. Les auteurs rapportent une étude rétrospective de 44 observations de LNH de l'anneau de Waldeyer colligées entre 1995 et 2002. Une chimiothérapie exclusive a été indiquée chez $80,6 \%$ des malades et une association chimio-radiothérapie a été réalisée chez 19,4\%. Le taux de survie globale était de $42 \%$ à 5 ans. Dans notre étude quatre facteurs de mauvais pronostic ont été individualisés : un âge z à 40 ans, les stades d'Ann Arbor II, III et IV, un taux de LDH $\geq 1,5$ fois la normale et la survenue de rechute.

Mots-clés : Lymphome, anneau de Waldeyer, chimiothérapie, radiothérapie, pronostic
\end{abstract}

Non Hodgkin's Lymphomas of the Waldeyer ring are a diverse group of neoplasms. They are particular in their clinic, therapy and prognostic. During the period between 1995 and 2002, 44 cases of non Hodgkin's lymphomas of the Waldeyer ring were reviewed. Chemotherapy was the exclusive treatment for $80,6 \%$ of patients and $19,4 \%$ were treated by chemo-radiotherapy. The total rate of survival within five years was $42 \%$. This rate was associated with age $\geq 40$ years old, Ann Arbor stage II, III and IV, serum lactate dehydrogenase

$(\mathrm{LDH}) \geq 1,5 \times$ normal value and relapse.

Keywords : Lymphoma, Waldeyer's ring, chemotherapy, radiotherapy, prognosis

\section{INTRODUCTION}

Les lymphomes non Hodgkiniens (LNH) sont des syndromes lymphoprolifératifs qui regroupent un grand nombre d'entités tumorales d'origine lymphoïde, de présentation clinique et de pronostic variables. Ils affectent habituellement les ganglions, mais dans 10 à $20 \%$ des cas des localisations extra-ganglionnaires sont associées (1). Les LNH de l'anneau de Waldeyer représentent plus du tiers de ces localisations extra-ganglionnaires $(2,3)$. Le traitement optimal de ces lymphomes reste controversé à cause du manque d'études randomisées et le regroupement hétérogène des différentes séries $(4,5)$.

L'objectif de notre travail est d'identifier les différents facteurs pronostiques de façon à discuter la meilleure attitude thérapeutique vis-à-vis de ces lymphomes agressifs.

\section{PATIENTS ET METHODES}

Notre étude est une étude rétrospective de 44 observations de LNH de l'anneau de Waldeyer qui ont été colligées à partir de registre du cancer du centre tunisien sur une période de 8 ans allant de 1995 à 2002. Les 44 dossiers de malades ont fait l'objet d'une description détaillée des caractéristiques épidémiologiques, cliniques, histologiques, thérapeutiques et évolutives. Dans tous les cas, le diagnostic a été porté sur des biopsies pratiquées au niveau du site tumoral. La classification histologique utilisée est celle de l'OMS 2002. Un bilan d'extension comportant une biopsie ostéo-médullaire, un scanner thoracoabdomino-pelvien et une endoscopie digestive a été réalisé pour tous les malades. On a classé nos malades selon la classification d'Ann Arbor.

La survie globale a été évaluée à 5 ans. Nous avons étudié l'influence de 8 facteurs pronostiques sur la survie. Toutes les données ont été saisies sur tableur Excel version 2003 et analysées à l'aide du logiciel statistique SPSS 11.5. La méthode de Kaplan-Meier était utilisée pour le calcul du taux de survie. L'étude comparative monofactorielle des éléments pronostiques était réalisée par le test statistique log-Ranke. La différence était considérée significative pour un $p \leq 0,05$.

\section{RESULTATS}

Sur le plan épidémiologique (Tableau I), il s'agissait de 29 hommes et 15 femmes avec un sex-ratio de 1,93. L'âge moyen de nos malades était de 54 ans avec des extrêmes de 6 et 87 ans. Dans notre série, une atteinte isolée de l'amygdale palatine a été notée dans $52 \%$ des cas, de l'amygdale pharyngienne dans $34 \%$ des cas, 
alors qu'une atteinte de l'amygdale linguale a été retrouvée dans $7 \%$ des cas. L'atteinte ganglionnaire était constante chez tous nos malades. Un taux de LDH sérique supérieur à 1,5 fois la normale a été trouvé chez $43,2 \%$ des cas. Pour la classification d'Ann Arbor, les stades localisés I et II étaient observés dans $63,7 \%$ des cas. Les formes d'architecture diffuse et les formes de haut grade de malignité sont le plus souvent observées. La variante à grandes cellules a été retrouvée dans $82 \%$ des cas et le lymphome de Burkitt a été diagnostiqué dans $11 \%$ des cas. Le phénotype $B$ était observé dans $84 \%$ des cas.

\begin{tabular}{|c|c|c|}
\hline & Nombre & Pourcentage \\
\hline \multirow[t]{2}{*}{ Sexe } & 29 & $66 \%$ \\
\hline & 15 & $34 \%$ \\
\hline Site tumoral Amygdale palatine & 23 & $52 \%$ \\
\hline Amygdale pharyngienne & 15 & $34 \%$ \\
\hline Amygdale linguale & 3 & $7 \%$ \\
\hline Multifocale & 3 & $7 \%$ \\
\hline Stade clinique d'Ann Arbor Stade I & 7 & $15,9 \%$ \\
\hline Stade II & 21 & $47,8 \%$ \\
\hline Stade III & 2 & $4,5 \%$ \\
\hline Stade IV & 14 & $31,8 \%$ \\
\hline Taux de LDH >1,5x normale & 19 & $43,2 \%$ \\
\hline \multirow[t]{2}{*}{ Histologie $\quad$ Phénotyp } & 37 & $84 \%$ \\
\hline & 7 & $16 \%$ \\
\hline
\end{tabular}

Tableau I : Caractéristiques cliniques des malades

Dans notre série, le traitement a été complété chez 36 malades, 3 malades sont décédés avant le début du traitement et 5 malades ont été perdus de vue. Une chimiothérapie exclusive a été indiquée pour $80,6 \%$ des malades et une association chimio-radiothérapie a été réalisée chez 7 malades $(19,4 \%)$. Ces derniers étaient tous classés stade I d'Ann Arbor. La dose moyenne qui a été délivrée au niveau de l'anneau de Waldeyer était de 44Gy. Plusieurs protocoles de chimiothérapie ont été utilisés CHVP (Cyclophosphamide, Hydroxyadriamycine, étoposide, Prednisone) ; CHOP (Cyclophosphamide, Hydroxyadriamycine, Oncovin, Prednisone) ; ACVBP (Adriblastine, Cyclophosphamide, Vindésine, Bléomycine, Prednisone) ; CEOP (Cyclophosphamide, Epirubicine, Vincristine, Prednisone) ; COPADEM (Cyclophosphamide, Vincristine, Prednisolone, Doxorubicine, Hydrocortisone, Acide folinique, Méthotrexate) (Tableau II).

\begin{tabular}{|c|c|c|}
\hline Protocole & Nombre & Pourcentage \\
\hline CHVP & 1 & $2,8 \%$ \\
CEOP & 4 & $11,1 \%$ \\
ACVBP & 4 & $11,1 \%$ \\
COP-COPADEM & 8 & $22,2 \%$ \\
Mini-CEOP & 9 & $25 \%$ \\
COP & 2 & $5,6 \%$ \\
CHOP & 7 & $19,4 \%$ \\
CVP & 1 & $2,8 \%$ \\
\hline Total & 36 & $100 \%$ \\
\hline
\end{tabular}

Tableau II : Les protocoles de chimiothérapie utilisés

Parmi les 36 malades ayant complété le traitement, 12 malades ont rechuté. Le délai moyen entre la fin du traitement et la rechute était de 20 mois avec des extrêmes de 3 à 72 mois.

Neuf malades sont décédés après un délai moyen de 7 mois, et 3 malades sont vivants en rechute. II y a eu un échec thérapeutique chez 10 malades qui sont tous décédés. Quatorze malades ont bien répondu au traitement et sont vivants en rémission complète après un recul de 11 ans. La durée moyenne de suivi des malades était de 34 mois, avec des extrêmes de 0 à 120 mois. Dans notre série, le taux de survie globale à 5 ans était de $42 \%$.

L'étude de l'influence des facteurs pronostiques sur la survie moyenne était comme suit concernant : (Tableau III) :

- L'âge: La moyenne de survie des malades âgés de moins de 40 ans était de 89 mois, alors qu'elle était de 40,6 mois pour les malades âgés de 40 ans ou plus ( $p=$ $0,01)$.

- Le sexe: La moyenne de survie des malades de sexe masculin était de 58,3 mois, alors qu'elle était de 40,4 mois pour les malades de sexe féminin $(p=0,49)$.

- Le phénotype: La survie moyenne pour le phénotype $B$ était de 57 mois, alors qu'elle était de 32,2 mois pour le phénotype $T(p=0,31)$.

- Le stade clinique d'Ann Arbor: La survie moyenne pour le stade I était de 71,6 mois, alors qu'elle était de 47,3 mois pour les stades II, III et IV associés $(p=0,05)$. - Le taux de lactate déshydrogénase (LDH): La survie moyenne pour les malades qui avaient un taux de LDH normal au moment du diagnostic était de 58,5 mois, alors qu'elle était de 33,4 mois pour ceux qui avaient un taux augmenté $(p=0,01)$.

- L'existence d'une atteinte médullaire initiale: La moyenne de survie des malades qui avaient une biopsie ostéo-médullaire normale était de 61,8 mois, alors qu'elle était de 38,8 mois pour les malades qui avaient un envahissement médullaire initial $(p=0,34)$.

- Le type de traitement: La survie moyenne des malades traités par chimiothérapie seule était de 60,8 mois, alors qu'elle était de 73,6 mois pour ceux traités par une association chimio-radiothérapie $(p=0,4)$. 
- La survenue d'une rechute: La survie moyenne des malades qui avaient présenté une rechute était de 38,2 mois, alors qu'elle était de 78,5 mois pour ceux qui n'avaient pas eu de rechute $(p=0,005)$.

\begin{tabular}{|lr|c|c|}
\hline \multicolumn{2}{|c|}{ Facteurs pronostiques } & $\begin{array}{c}\text { Survie moyenne } \\
\text { en mois }\end{array}$ & P \\
\hline Age & Age $<40$ ans & 89 & \\
& Age $\geq 40$ ans & 40,6 & 0,01 \\
\hline Sexe & Masculin & 58,3 & \\
& Féminin & 40,4 & 0,49 \\
\hline Phénotype & $\mathrm{B}$ & 57 & \\
& $\mathrm{~T}$ & 32,2 & 0,31 \\
\hline Stade clinique & Stade I & 71,6 & \\
& Stade II, III, IV & 47,3 & 0,05 \\
\hline Taux de LDH & Normal & 58,5 & \\
& Augmenté & 33,4 & 0,01 \\
\hline Envahissement & Absent & 61,8 & \multirow{2}{*}{ Médullaire } \\
& Présent & 38,8 & 0,34 \\
\hline Type du traitement & CT seule & 60,8 & 0 \\
& CT-RT & 73,6 & 0,4 \\
\hline Rechute & Absente & 78,5 & 0,005 \\
& Présente & 38,2 & 0,01 \\
\hline
\end{tabular}

Tableau III: Influence des facteurs pronostiques sur la survie

Ainsi, notre étude statistique n'a retenu que 4 facteurs de mauvais pronostic : un âge supérieur ou égal à 40 ans, les stades cliniques d'Ann Arbor II, III et IV, un taux de $\mathrm{LDH} \geq 1,5$ fois la normale et la survenue d'une rechute.

\section{DISCUSSION}

Les lymphomes cervico-faciaux représentent 10 à 30\% des LNH extra-ganglionnaires $(6,7)$ dont 20 à $30 \%$ sont localisés au niveau de l'anneau de Waldeyer $(2,3)$ qui représente la 2éme localisation extra-ganglionnaire des LNH après la localisation digestive et le premier site des LNH cervico-faciaux $(4,8)$.

Le traitement des LNH cervico-faciaux a longtemps reposé sur la radiothérapie exclusive à la dose de 30 à 50 Gy avec un excellent taux de contrôle local. Vu le risque de rechute élevé d'environ 40 à $50 \%$ observé avec cette modalité, l'utilisation d'un traitement associant une chimiothérapie et une radiothérapie a été préconisée dans les stades localisés (4, 9). Avilés (4), a rapporté une étude prospective randomisée portant sur 316 malades ayant un LNH de l'anneau de Waldeyer classés stade I selon la classification d'Ann Arbor, les malades ont été divisés en 3 groupes : le 1er groupe a été traité par une radiothérapie $(R T)$ seule $(n=108)$, le 2ème groupe a eu une chimiothérapie (CT) seule $(n=122)$ et pour le 3ème groupe, une chimio-radiothérapie (CT-RT) a été préconisée. Une rémission complète a été observée dans $93 \%$ des cas pour le 1er groupe, dans $87 \%$ pour le 2ème groupe et dans $97 \%$ des cas pour le dernier groupe. La différence entre les 3 groupes n'était pas significative en matière de rémission complète, mais les rechutes étaient plus fréquentes pour les malades traités soit par RT ou CT seule et la survie à 5 ans était de $51 \%$ pour la CT seule, $56 \%$ pour la RT seule : ces résultats sont statistiquement différents de la survie à $90 \%$ obtenue par association RT- CT $(p<0,001)$. La CT permet d'assurer une prévention plus efficace des rechutes à distance, notamment des rechutes gastriques particulièrement fréquentes dans les localisations primitives de l'anneau de Waldeyer $(10,11)$. Les protocoles utilisés (12) comprennent habituellement plusieurs médicaments, la plupart des associations comprennent de la doxorubicine et /ou du cyclophosphamide. Le protocole le plus connu étant le protocole $\mathrm{CHOP}$ avec lequel le pourcentage de rémission complète est de l'ordre de $70 \%$ (12). D'autres protocoles ne contenant pas la doxorubicine sont néanmoins actifs, à condition d'être donnés à doses suffisantes. La RT était la modalité thérapeutique la plus fréquemment utilisée pour les lymphomes extra-ganglionnaires $(13,14)$, mais, des études récentes ont montré que la modalité combinant une RT à une CT, offre le meilleur résultat pour la survie $(4,6,15,16)$. Dans tous les cas, le choix du traitement dépend aussi du sous-type histologique (17), ce qui explique l'utilisation la plus fréquente de la CT soit seule ou après RT dans les lymphomes diffus à grandes cellules. Actuellement, on considère que l'association RT et CT selon différentes modalités est le traitement le plus approprié des LNH stades I et II de l'anneau de Waldeyer $(4,17,18,19)$, et les études faites dans ce sens ont conclu à la supériorité de cette modalité par rapport à une CT seule ou une RT seule. Pour notre série $19,4 \%$ des malades ont été traités par une association CT-RT, alors que $80,6 \%$ des malades ont été traités par une CT seule. Pour les LNH de l'anneau de Waldeyer, la survie globale à 5 ans varie de $93 \%$ [20] à $100 \%$ (17), et la survie sans rechute est de $80 \%$ à 5 ans $(20,21)$. Pour notre série, le taux de survie globale à 5 ans était de $42 \%$, un taux inférieur à ceux rapportés dans la littérature. Ce taux peut être expliqué par le fait que $57 \%$ de nos malades étaient âgés de plus de 60 ans et que 37,3\% d'entre eux étaient classés stade III ou IV d'Ann Arbor. Le taux de survie est corrélé à plusieurs facteurs pronostiques différents selon les auteurs (Tableau IV).

\begin{tabular}{|c|c|c|c|c|c|c|}
\hline Auteurs & Age & Histologie & $\begin{array}{c}\text { Ann } \\
\text { Arbor }\end{array}$ & $\begin{array}{c}\text { Nbre de } \\
\text { sites }\end{array}$ & TNM & $\begin{array}{c}\text { Taille } \\
\text { tumorale }\end{array}$ \\
\hline Conley [22] & $\mathrm{NE}$ & + & $\mathrm{NE}$ & $\mathrm{NE}$ & $\mathrm{NE}$ & - \\
\hline Goldwein[23] & - & - & - & + & $\mathrm{NE}$ & + \\
\hline Jacobs [24] & $\mathrm{NE}$ & + & + & $\mathrm{NE}$ & - & - \\
\hline Notre série & + & - & + & $\mathrm{NE}$ & $\mathrm{NE}$ & $\mathrm{NE}$ \\
\hline
\end{tabular}

$(\mathrm{NE}=$ facteur non étudié) $\quad(+)$ : différence significative $(-)$ : pas de différence significative

Tableau IV: Facteurs pronostiques selon la littérature 
Dans notre étude, l'âge supérieur à 40 ans a constitué un facteur de mauvais pronostic pour nos malades, ceci est conforme aux données de la littérature. En effet, Barton [8] a montré une survie à 5 ans meilleure pour les malades de moins de 40 ans (86\%) que pour les malades d'âge $\geq$ à 40 ans (49\%). Cette différence ne devient significative qu'à partir de l'âge de 60 ans pour la plupart des auteurs (18, 25, 26, 27). Pour Banfi (28) et Economopoulos (29), l'âge ne constitue pas un facteur pronostique.

Le sexe ne constitue pas un facteur pronostique pour la quasi-totalité des auteurs $(28,30)$, comme c'était le cas pour notre série. En revanche, pour Mohommadianpanah (1) et Oguichi (27) le sexe masculin constitue un facteur de mauvais pronostic pour les LNH amygdaliens. Une « performance status $\gg \geq 2$, évaluée selon le code ECOG ou OMS, constitue un facteur de mauvais pronostic pour Yong (14) et Oguichi (27). En revanche, Ezzat (13) et Mohammadianpanah (1) soulignent qu'un état général entre 0 et 1 constitue un facteur de bon pronostic. Dans notre étude, la survie en fonction de l'état général du malade n'a pas été évaluée car on ne disposait pas d'une évaluation de l'état général des malades selon le code ECOG ou la classification OMS.

Certaines études ont conclu que la présence des symptômes $\mathrm{B}$ (amaigrissement $\geq$ à $10 \%$ du poids du corps, fièvre $\geq$ à $38^{\circ}$ pendant 15 jours et des sueurs nocturnes abondantes) constitue un élément péjoratif pour ces lymphomes $(14,26,31,32)$.

L'histologie est déterminante pour Pasini (33) et Yong (14). Pour eux, les lymphomes diffus sont de moins bon pronostic que les lymphomes de bas grade et de grade intermédiaire. De plus, les lymphomes indifférenciés et les lymphomes de haut index mitotique sont péjoratifs $(20,34)$. Pour Mtasiwa (35), I'histologie constitue un facteur pronostique indépendant des autres facteurs et doit être intégrée dans l'index pronostique international (IPI). En revanche pour Liang, ce facteur ne peut être considéré indépendamment du stade clinique et l'âge (32). Alors que la majorité des auteurs s'accordent sur le fait que le haut grade histologique constitue un facteur de mauvais pronostic $(14,33,35)$, certains d'entre eux trouvent que I'histologie ne constitue pas un facteur prédictif de l'évolution des LNH (30). Pour certains auteurs, le phénotype $\mathrm{B}$ constitue un facteur de bon pronostic (36) alors que le phénotype $\mathrm{T}$ constitue un facteur péjoratif pour les $\mathrm{LNH}$ de l'anneau de Waldeyer $(31,34,36)$. Le phénotype du lymphome n'était pas un facteur pronostique pour notre série.

Du point de vue site tumoral initial, la localisation au niveau de l'amygdale palatine a un meilleur pronostic que pour la base de langue et le nasopharynx $(9,13)$. Pour Banfi (28), le pronostic des LNH touchant la base de langue et l'amygdale pharyngienne, parait meilleur à celui des LNH des amygdales palatines : survie à 5 ans de
$50 \%$ versus $30 \%$. Economopoulos (29) n'a pas trouvé de différence significative entre les différentes structures de l'anneau de Waldeyer. Pour Bajetta (21), la survie des LNH de l'anneau de Waldeyer dépend des autres facteurs et non du site tumoral.

Le stade clinique d'Ann Arbor constitue un facteur pronostique déterminant pour les LNH de l'anneau de Waldeyer (7). Pour Jacobs (24), la survie pour les histologies défavorables est fortement influencée par le stade clinique, la survie à 5 ans est de $48 \%$ pour les stades I et $35 \%$ pour les stades II. De même pour Endo (18), la survie à 5 ans était de $100 \%$ pour le stade I et de $32,4 \%$ pour le stade II. Pour notre série, la survie à 5 ans était de $83 \%$ pour le stade I, $42 \%$ pour le stade II. Jacobs (24) n'a trouvé aucune valeur pronostique de la classification TNM pour les LNH extra-ganglionnaires. Kobayachi (26), qui a classé 74 malades ayant un LNH de l'anneau de Waldeyer selon la classification TNM a conclu que les stades I et II ont un meilleur pronostic que les stades III et IV. Les malades classés N0 avaient un taux de survie à 5 ans de $93 \%$ versus $0 \%$ pour les malades classés N3.

Le type du traitement ainsi que les modalités thérapeutiques constituent, eux aussi, un facteur pronostique surtout pour les lymphomes localisés $(37,38)$. Un traitement associant une CT à une RT pour les stades localisés donne un taux de survie supérieur à la RT seule ou une CT seule $(1,37)$, vu le risque des rechutes très important après RT seule. Dans notre série, la modalité de traitement (RT, CT-RT) n'avait pas d'influence sur la survie de nos malades.

Plusieurs facteurs biologiques ont été étudiés dans la littérature comme facteurs pronostiques dans les LNH de l'anneau de Waldeyer. Parmi ces facteurs : le taux de LDH qui représente actuellement un facteur pronostic essentiel au même titre que l'âge, l'extension tumorale et l'état général (26). Medrano (39) a conclu qu'un taux de LDH élevé constitue un facteur pronostique statistiquement significatif $(p=0,001)$. Pour notre série, un taux de $\mathrm{LDH} \geq$ à 1,5 fois la normale a constitué un facteur péjoratif. La 32 microglobuline sérique, qui a une valeur pronostique majeure dans les lymphomes diffus de haut grade de malignité (40), n'a pas été étudiée dans les LNH localisés au niveau de l'anneau de Waldeyer. L'étude la plus récente est celle de Medrano (39) qui a trouvé que le taux de 32 microglobuline constitue un facteur pronostique statistiquement significatif ( $p=0,02$ ) pour les lymphomes cervico-faciaux d'une façon générale. Des études récentes ont montré aussi que l'anticorps monoclonal ki-67, marqueur de prolifération cellulaire, pourrait représenter un nouvel indicateur de pronostic et permettre ainsi de moduler les indications de chimiothérapie. En effet, une équipe japonaise [29] a montré que la médiane de survie est meilleure quand il existe peu de cellules ki-67+ à la fois dans les lymphomes B et les lymphomes T. La protéine bcl6, exprimée par les centrocytes 
et les centroblastes de phénotype B dans le tissu lymphoïde normal, est actuellement mise en évidence dans les LNH des amygdales (41). L'expression de la bcl6 constitue un facteur de bon pronostic $(p=0,049)$ pour Weng (42) et Ree (41).

L'index pronostique international IPI $\leq 2$ ou $>2$, incluant 5 facteurs: l'âge, l'indice d'activité, le stade d'Ann Arbor, le taux LDH et le nombre de localisation extra-ganglionnaire, constitue pour certains, le facteur pronostique le plus important dans le cadre des LNH de l'anneau de Waldeyer (8). Certaines études ont toutefois estimé que cet IPI, initialement élaboré pour évaluer le pronostic des LNH à localisation ganglionnaire, est peu adapté au LNH de l'anneau de Waldeyer. Ils ont même proposé l'élaboration d'un nouvel IPI tenant compte de la taille tumorale, du siège et de la taille des adénopathies associées (13). Certaines études ont montré une corrélation entre la présence de l'EBV dans les lymphomes (détecté par hybridation in situ) et le pronostic. En analyse univariée, cette donnée est associée pour la survie globale, avec l'âge, I'IPI et le taux de LDH. En analyse multivariée, la présence de l'EBV interagit avec l'âge et pour seuls les malades de plus de 60 ans en découle un pronostic péjoratif. Enfin, en couplant EBV et IPI, seule la présence de l'EBV reste un facteur indépendant pour diminuer la survie (38). L'identification des facteurs pronostiques constitue le «gold standard» dans le choix de la modalité thérapeutique surtout pour les formes localisées de LNH. En Tunisie, le traitement est basé sur le protocole national du traitement des lymphomes agressifs, élaboré en 2002 par le groupe d'étude des lymphomes en Tunisie (GELT). Dans le protocole quatre facteurs pronostiques ont été pris en considération : Age ( $<70$ ans ; $>70$ ans), le taux LDH, le stade d'Ann Arbor et le PS >2.

\section{CONCLUSION}

Le traitement des LNH de l'anneau de Waldeyer est jusqu'à nos jours un sujet de controverse. Mais, on tend actuellement à considérer que l'association radiothérapie - chimiothérapie est le traitement le plus approprié des stades localisés de ces lymphomes. Différents facteurs pronostiques ont été étudiés dans la littérature. Notre analyse statistique n'a pu retenir que 4 facteurs de mauvais pronostic : un âge $\geq$ à 40 ans, les stades cliniques II, III et IV, un taux de $\mathrm{LDH} \geq 1,5$ fois la normale et la survenue d'une rechute. La recherche des facteurs pronostiques est indispensable dans le bilan pré thérapeutique des LNH. Leur identification permet d'indiquer la modalité thérapeutique la plus appropriée (RT, CT-RT) ainsi que le protocole de chimiothérapie optimal.

\section{REFERENCES}

1-Mohammadinpanah M, Omidavai S, Mosalei A, Ahmadloo N. Treatment results of tonsillar lymphoma: a 10 years experience. Ann Hematol 2005; 84(4): 223-6

2- Lee JH, Lee SS, Park JH, Kim YW, Yang MH. Prevalence of EBV RNA in sinonasl and waldeyer's ring lymphomas. J Korean Med Sci 1994; 9 (4): 281 - 8 3-Zhou J, Wei L, Cao Y. Analysis on clinical and immunohistochemical characteristics of primary extranodular non Hodgkin's lymphoma in otolaryngol head and neck. Lin Chuang Er Yan Hou Ke Za Zhi 2003; 17 (12); 724 - 6 4-Avilés A, Delgado S, Ruiz H, De la Torre A, Guzman R, Talavera A. Treatment of non-Hodgkin's lymphoma of Waldeyer's ring: Radiotherpy versus chemotherapy versus combined therapy. Eur J Cancer B Oral Oncol 1996; 32 (1): 19 - 23 5- Endo S, Kida A, Sawada U et al. Clinical anlysis of malignant lymphoma of the tonsil. Acta Otolaryngol 1996; 523: 263-6

6- Economopoulos T, Fountzilas G, Kostourou A et al. Primary extranodal nonHodgkin's lymphoma of the head and neck in adults : a clinicopathological comparaison between tonsillar and non tonsillar lymphoma. Anti cancer Res 1998; 18(6B): 4655 - 60

7- Hanna E, Wanamaker J, Adelstein D, Tubbs R, Lavertu P. Extranodal lymphoma of the head and neck: a 20 years experience. Arch Otolaryngol Head
Neck Surgery 1997; 123 (12): 1318 - 23

8- Barton JH, Osborne BM, Butler JJ et al. Non-Hodgkin's lymphoma of the tonsil: a clinico-pathologic study of 65 cases. Cancer 1984; 53 (1): 86 - 95

9- Nishioka T, Tsuchia K, Nishioka S et al. Pilot study of modified version of CHOP puls radiotherapy for early stage agressive non Hodgkin's lymphoma of the head and neck. Int J Rad Oncol Biol Phys 2004; 60(3): 847-52

10- Cosset JM, Ozanne F, Henry-amar M et al. An alternating chemotherapy and radiotherapy combination for non-Hodgkin's lymphoma of unfavorables histologies: Feasibility and prelimenary results. Radiother Oncol 1985; 3 (2): 133-8 11- Jacobs C, Weiss L, Hoppe RT. The managment of extranodal head and neck lymphomas. Arch Otolaryngol Head Neck Surg 1986; 112 (6): 654- 8

12- Hiramatsu $\mathrm{H}$, Kondo M, Uematsu M, Shigmatsu N, Ando $\mathrm{Y}$, Hashimoto $\mathrm{S}$. Non- Hodgkin's lymphoma of Waldeyer's ring: the treatment results of the past 22 years and the signifiance of CHOP. Nippon Gan Chiryo Gakkai Shi 1990; 25 (10): 2477- 83

13- Ezzat AA, Ibrahim EM, El Weshi AN et al. Localized non-Hodgkin's lymphoma of Waldeyer's ring: clinical features, management, and prognosis of 130 adults patients. Head Neck. 2001; 23 (7): 547- 58 
14- Yong W, Zhang Y, Zheng W, Wei Y. Prognostic factors and therapeutic efficacy and combined radio-chemotherapy in Waldeyer's ring non-Hodgkin lymphoma. Chin Med J (Engl) 2000; 113 (2): 148- 50

15- Hatta C, Ogasawara H, Okita J et al. Non Hodgkin's malignant lymphoma of sinonasal tract: treatment outcome for 53 patients according to Real classification. Auris Nasus Larynx 2001; 28(1): 55- 60

16- Lei KI, Suen JJ, Hui P et al. Primary nasal and nasopharyngeal lymphomas : a comparative study of clinical presentation and treatment outcome. Clin Oncol 1999; 11(6): 379-87

17- Hart S, Horsman JM, Radston CR, Hanckock H, Goepel JR, Hanckock BW. Localised extranodal lymphoma of the head and neck: The Sheffield lymphoma Group experience(1971-2000). Clin Oncol2004; 16(3): 186-92

18- - Endo S, Kida A, Sawada U et al. Clinical anlysis of malignant lymphoma of the tonsil. Acta Otolaryngol 1996; 523: 263-6

19- Gurkaynak M, Gengiz M, Akyurek S, Ozyar E, Atham IL, TekuzmanG. Waldeyer's ring lymphomas: treatment results and prognostic factors. Am J Clin Oncol 2003; 26(5): 437-40

20- Plantenga KF, Hart G, Van Heerde P, Tierie AH. Non-Hodgkin's malignant lymphoma of upper digestive and respiratory tracts. Int $\mathrm{J}$ Radiol Oncol Biol Phys 1981; 7(10): 1419- 27

21- Bajetta E, Buzzoni R, Rille F et al. Non-Hodgkin's lymphoma of Waldeyer's ring. Tumori 1983; 69(2): 129-36

22- Conley SF, Staszak C, Clammon GH, Maves M. Non-Hodgkin's lymphoma of the head and neck: the university of Lowa experience. Laryngoscope 1987; 97 (3): $291-300$

23- Goldwein JW, Coia LR, Hankes GE. Prognostic factors in patients with early stage non-Hodgkin's lymphoma of the head and neck treated definitive radiation. Int J Radiat Oncol Biol Phys 1991; 20(1): 45-51

24- Jacobs C, Hoppe RT. Non Hodgkin's lymphoma of the head and neck extranodal sites. Int J Radiat Oncol Biol Phys 1985; 11(2): 357-64

25- Frata P, Buglione M, Grisanti S, Bonetti B et al. Localized extranodal lymphoma of the head and neck : retrospective analysis of a series of 107 patients from a single institution. Tumori 2005; 91(6): 456-62

26- Kobayashi Y, Ogino T, Hayashi T, Nonaka S, Harabuchi Y. Prognostic factors in non-Hodgkin's lymphoma of Waldeyer's ring and the lymph nodes of the neck. Nippon Jibiinkoka Gakkai Keiho 2000; 103(6): 761-9

27- Oguchi $\mathrm{O}$, Ikeda $\mathrm{H}$, Isobe $\mathrm{K}$ et al. Tumor bulk as a prognostic factor for the management of localized aggressive non Hodgkin's lymphomas: A survey of the Japan Lymphoma Radiation Therapy Group. Int J Rad Oncol Biol Phys 2000; 48(1): 161-8

28- Banfi A, Bonadonna G, Ricci SB et al. Malignant lymphoma of Waldeyer's ring: Natural history and survival after radiotherapy. Br Med J 1972; 3(5819); $140-3$
29- Economopoulos $\mathrm{T}$, Asprou N, Stathakis $\mathrm{N}$ et al. Primary extranodal non Hodgkin's lymphoma of the head and neck. Oncology 1992; 49(6): 484-8 30- Brugère J, Schellienger M, Gérald-Marchant R, Tubiana M, Pouillart P, Cachin Y. Non-Hodgkin malignant lymphomata of upper digestive and respiratory tract: natural history and results of radiotherapy. $\mathrm{Br} \mathrm{J}$ Cancer 1975; 31(2): $435-40$

31- Armitage JO, Vose JM, Linder $\mathrm{J}$ et al. Clinical signifiance of immunophenotype in diffuse aggressive non-Hodgkin's lymphoma. J Clin Oncol 1989; 7(12): $1783-90$

32- Ling R, Todd D, Ho FC. Aggressive non Hodgkin's lymphoma: T-cell versus B-cell. Hematol Oncol $1996 ; 14(1): 1-6$

33- Pasini F, Cetto GL, Todeschini G et al. Combination of chemotherapy and radiotherapy improves the cure rate in primary extranodal lymphomas of the head and neck. Anticancer Res 1997; 17(4A): 2837-42

34- Yamanaka N, Hrabuchi Y, Kataura A. The prognostic value of KI-67 antigen in non-Hodgkin lymphoma of Waldeyer's ring and nasal cavity. Cancer 1992; 70(9): 2342-9

35- Mtasiwa DM, Imamura N, Inda T et al. Immunophenotype, histology and clinical stage: their predictive value in the prognosis of non Hodgkin's lymphomas. Hiroshima J Med Sci 1990; 39(4): 95-102

36- Hutchison RE, Berard CW, Shuster JJ, Link MP, Pik TE, Murphy SB; B-cell lineage confers a favorable outcome among children and adolescents with large-cell lymphoma: a Pediatric Oncology Group Study. J Clin Oncol 1995; 13(8): 2023-32

37- Ling R, Chiou E, Todd D, Chan TK, Choy D, Loke SL. Combined chemotherapy and radiotherapy for lymphomas of Waldeyer's ring. Oncology 1991; 48 (5): $362-4$

38- Munck JN, Dhermain F, Koscienly S et al. Alternating chemotherapy and radiotherapy for limited-stage intermediaire and high-grade non-Hodgkin's lymphomas: long-term results for 96 patients with tumors $>5 \mathrm{~cm}$. Ann Oncol 1996; 7(9): 925-31

39- Medrano Vera ML. Situation for hed and neck lymphomas in Chile. Otolaryngol Head Neck Surg 2004; 131(2): 311-20

40- Lopez-Gullermo A, Colomo L, Jimenez $\mathrm{M}$ et al. Diffuse large B-cell lymphoma: clinical and biological characterization and outcome according to the nodal or extranodal primary origin. J Clin Oncol 2005; 23(12): 2797-804

41- Ree HJ, Ohsima K, Aozasa K et al. Detection of germinal center B-cell lymphoma in archival specimens: critical evaluation of Bcl6 protein expression in Diffuse large B-cell lymphoma of the tonsil. Human Pathol 2003; 34(6): 610- 6 42- Weng Y, Gao ZF, Liu K, Zhang WJ, Ke XY, Li M. Factors affecting the prognosis of diffuse large B-cell lymphoma in Chinese. Zhonghua Nei Ke Za Zhi 2005; 44(9): 681-3 